\title{
Satisfaction With Family Life Scale: Adaptação e Evidências de Validade e Precisão
}

\author{
Luize Anny Guimarães Amorim¹ (D), Patrícia Nunes da Fonsêca (D) \\ Universidade Federal da Paraíba, João Pessoa-PB, Brasil
}

RESUMO

A pesquisa objetivou adaptar a escala Satisfaction with Family Life (SWFL) para o português-brasileiro, reunindo evidências de validade fatorial e consistência interna. No Estudo 1, participaram 216 pessoas, com idades entre 18 e 92 anos $(M=38,43 ; D P=15,90)$, sendo 71,6\% mulheres, que responderam ao SWFL a e questões sociodemográficas. A análise fatorial exploratória reportou uma estrutura unifatorial com 75\% de variância total explicada e um alfa de Cronbach de 0,89. No Estudo 2, a amostra foi de 214 pessoas, com idades entre 18 e 77 anos $(M=29,8 ; D P=11,65)$, a maioria mulheres $(69,6 \%)$, que responderam aos mesmos instrumentos do Estudo 1 . Os achados da análise fatorial confirmatória asseveraram a estrutura unifatorial, apresentando um alfa de Cronbach de 0,89 . Conclui-se que o instrumento apresenta parâmetros psicométricos adequados e poderá ser utilizado em futuras pesquisas sobre família.

Palavras-chave: testes psicológicos; satisfação; família.

\section{ABSTRACT - Satisfaction with Family Life Scale: Adaptation and Evidence of Validity and Reliability}

This study aimed to adapt the Satisfaction with Family Life Scale (SWFLS) to Brazilian Portuguese, supported by factorial validity and internal consistency evidence. Study 1 included 216 respondents, aged between 18 and 92 years $(M=38.43 ; S D=15.90)$, of whom $71.6 \%$ were female, who answered to the SWFLS and sociodemographic questions. The exploratory factorial analysis indicated a one-factor structure with $70 \%$ of Total Variance Explained and a Cronbach's alpha of .89. In Study 2, the sample was composed of 214 respondents, aged between 18 and 77 years $(M=29.8 ; S D=11.65)$, the majority female $(69.6 \%)$, who answered the same instruments as Study 1. The Confirmatory Factor Analysis confirmed a single-factor structure, presenting a Cronbach's alpha of .89. It was concluded that the instrument has adequate psychometric properties, representing a valuable tool for use in future research with families.

Keywords: psychological testing; satisfaction; family.

\section{RESUMEN - Satisfaction With Family Life Scale: Adaptación y Evidencias de Validez y Confiabilidad}

La investigación tuvo como objetivo adaptar la Satisfaction with Family Life Scale (SWFLS) al portugués brasileño, reuniendo evidencias de validez factorial y consistencia interna. En el Estudio 1, participaron 216 personas, con edades entre 18 y 92 años $(\mathrm{M}=38,43$, DS $=15,90)$, siendo $71,6 \%$ mujeres, que respondieron al SWFLS y cuestiones sociodemográficas. El análisis factorial exploratorio reportó una estructura unifactorial con un $75 \%$ de varianza total explicada y un alfa de Cronbach de 0,89 . En el Estudio 2 , la muestra fue de 214 personas, con edades entre 18 y 77 años $(M=29,8, D S=11,65)$, la mayoría mujeres $(69,6 \%)$, que respondieron a los mismos instrumentos del Estudio 1. Los hallazgos del análisis factorial confirmatorio asistieron a la estructura unifactorial, presentando un alfa de Cronbach de 0,89 . Se concluye que el instrumento presenta parámetros psicométricos adecuados, y podrá ser utilizado en futuras investigaciones sobre familia.

Palabras clave: tests psicológicos; satisfacción; familia.

A família vem passado por inúmeras mudanças nas últimas décadas, fato é que não há mais um único modelo familiar, mas uma diversidade de arranjos que trazem consigo consideráveis desafios para seus membros (Walsh, 2016). Assim, na tentativa de entender as dificuldades e fortalecer a unidade familiar, os pesquisadores têm buscado estudar os aspectos positivos da vida familiar, a exemplo da satisfação (Grilo, \& Major, 2015; Silva et al., 2017).
No presente estudo, busca-se pesquisar a satisfação familiar, visto ser um contexto relevante na constituição e formação do ser humano, além de ser a base da sociedade (Brasil, 1988). Ademais, poder-se-ia identificar os aspectos positivos da família, ou seja, aqueles que geram prazer e alegria, bem como os fatores que proporcionam o desenvolvimento psicológico sadio do ser humano, suas competências e os motivos que os instigam a conquista da felicidade (Seligman, \& Csikszentmihalyi, 2000).

${ }^{1}$ Endereço para correspondência: Rua Adalgisa Carneiro Cavalcanti-777, Cuiá, apto 201, Bloco D, 58077-274, João Pessoa, PB. E-mail: luizeannyamorim@gmail.com O artigo é derivado da Tese de doutorado da Luize Anny Guimarães Amorim com orientação de Patrícia Nunes da Fonseca, defendida em 2021 no programa de pós-graduação em Psicologia Social da Universidade Federal da Paraíba, João Pessoa-PB, Brasil. 
Tais aspectos são estudados pela Psicologia Positiva com vistas à prevenção e promoção da saúde mental. Ademais, essa perspectiva não nega os aspectos negativos dos seres humanos, todavia, busca investigar, compreender e valorizar as potencialidades humanas, enfatizando as capacidades e motivações (Weiting, 2017).

Portanto, salienta-se a necessidade de se compreender como as pessoas avaliam suas vidas em família, ou seja, o quanto elas são satisfeitas com o seu contexto familiar, visto que tal avaliação poderá interferir diretamente na qualidade de vida das pessoas, na saúde física e psicológica e no enfrentamento do estresse (Zabriskie, \& McCormick, 2003).

Pelo exposto, percebe-se a pertinência de conhecer os aspectos que compõem a satisfação familiar e, para isso, é necessário que se tenham instrumentos adequados no contexto brasileiro para mensurar tal construto. Logo, a fim de localizar possíveis medidas, foi realizado um levantamento de publicações de cunho psicométrico nas bases de dados do SciELO (Scientific Eletronic Library Online), PePSIC, Index Psi, e PsycINFO no dia 23 de junho de 2020, sem limitação de tempo (considerou-se todos os anos), com o objetivo de verificar a existência de instrumentos brasileiros ou adaptados para o Brasil que mensurassem a satisfação familiar. Foram utilizados os termos "escala" e "satisfação familiar" ou "satisfação com a vida familiar", mas não foi localizada nenhuma publicação de natureza psicométrica, seja de adaptação ou validação de instrumentos direcionados à avaliação específica da satisfação familiar. Diante desse resultado, percebeu-se a necessidade de se ter um instrumento que mensurasse esse construto na realidade brasileira com índices psicométricos adequados. Então, o presente estudo teve por objetivo adaptar a Satisfaction with Family Life Scale (SWFLS) para o português-brasileiro, reunindo evidências de sua validade fatorial e consistência interna.

A seguir será abordada a conceituação da satisfação familiar e, em seguida, serão apresentadas as medidas construídas em âmbito nacional e internacional no que tange ao construto em questão.

\section{Satisfação Familiar}

A satisfação familiar é um construto que tem sido pouco estudado, em detrimento de outros temas relacionados à família, a exemplo da satisfação parental e conjugal (Scorsolini-Comin et al., 2015; Scorsolini-Comin et al., 2016). Sua elaboração é extraída do conceito de satisfação com a vida (Shin, \& Johnson, 1978), a qual se refere a parte cognitiva do bem-estar subjetivo.

Nessa ocasião, será enfatizada a avaliação de uma determinada área da vida, a família. Para tal julgamento, o indivíduo deverá se basear nos próprios critérios, ou seja, nos padrões que estabeleceu no decorrer da vida. Assim, esse julgamento é considerado satisfatório quando o indivíduo, por meio de um processo comparativo, assume que sua vida com sua família segue o padrão que ele julga ser o ideal (Pavot, \& Diener, 2008).

Essa perspectiva mais global de avaliação permite que o sujeito faça uma reflexão mais ampla, considerando diferentes domínios de sua vida familiar, e baseando-se no seu próprio conjunto de valores. Ademais, ao fazer tal julgamento, as pessoas harmonizam seus sentimentos, anseios, frustrações, aspectos contextuais e desejos, estabelecendo, portanto, seu nível de satisfação familiar (Ward et al., 2009).

Lisboa et al. (2014) apontam que a satisfação com a família é um fator de proteção para adolescentes, especialmente quanto ao envolvimento em situações de vitimização e agressão entre pares, pois o acolhimento dos pais auxilia na prevenção da exposição desses indivíduos a situações de vulnerabilidade. Ademais, Zabriskie e McCormick (2003) apontam que a satisfação familiar e as atividades de lazer em família têm uma relação positiva.

\section{Mensuração da Satisfação Familiar}

Os primeiros instrumentos elaborados para avaliação da satisfação familiar datam da década de 70 (Andrews, \& Withey, 1976; Campbell et al., 1976), tendo como um dos pioneiros o Family Life Questionnaire (Guerney, 1977, conforme citado em Zabriskie, \& Ward, 2013). Com o passar dos anos, foram surgindo novas medidas de avaliação do contexto familiar, a exemplo da Family Adaptability and Cohesion Evaluation Scale (FACES) (Olson et al., 1985), baseadas no Modelo Circumplexo de Sistemas Familiares e Conjungais de Olson (1979). As três primeiras versões da FACES, conforme apontam seus autores, não avaliam de modo preciso os níveis mais extremos das dimensões Coesão e Flexibilidade. Sendo assim, foi elaborada uma nova versão (FACES IV) a fim de melhor avaliá-las.

A FACES IV é composta por 62 itens, sendo 42 subdivididos em duas subescalas equilibradas (Coesão e Flexibilidade) e quatro subescalas desequilibradas (Desengajada, Emaranhada, Caótica e Rígida), cada uma contendo sete itens. Além dessas, há as subescalas de comunicação e de satisfação (dez itens, cada). A medida apresentou boas qualidades psicométricas, incluindo validade de construto e concorrente (Olson, 2011). Recentemente, foi realizada uma adaptação semântica dessa medida para o Brasil, mas sem análises psicométricas (Santos et al., 2017). Vale salientar que esse instrumento não mensura, de modo específico, a satisfação com a família em si, mas a adaptabilidade e coesão dela.

Baseado no mesmo modelo teórico das escalas supracitadas, foi construída a Family Satisfaction Scale (FSS) com 14 itens (Olson, \& Wilson, 1982). Essa medida passou a ser uma das mais aceitas e largamente utilizadas para avaliar esse construto. Atualmente, foi reduzida a 10 itens que avaliam a satisfação familiar em termos de coesão, flexibilidade e comunicação, e tem demonstrado boas evidências de validade e precisão (Olson, 2004; 2011). 
Apesar disso, essa medida foi questionada por pesquisadores como sendo uma que não avalia especificamente satisfação familiar, mas outros construtos, a exemplo da coesão (Schumm et al., 1986).

Outra escala conhecida é a Kansas Family Life Satisfaction Questionnaire (McCollum et al., 1988), a qual avalia a satisfação da família perguntando, em quatro itens, quão satisfeitas ou insatisfeitas as pessoas se encontram em relações específicas, tais como relacionamento conjugal, parental, fraternal e familiar de forma global. Uma de suas críticas é a limitação quanto à aplicação, pois só pode ocorrer em famílias com quatro ou mais membros, dentre eles, um casal e, ao menos, dois filhos. Outro ponto é a existência de apenas um item relacionado à satisfação familiar global, ou seja, uma questão direta que busca saber o quão satisfeita a pessoa está com sua família. Segundo os autores, esse item parece estar desassociado dos demais que mensuram construtos independentes, a saber: a satisfação conjugal e parental (Schumm et al., 1986).

Um instrumento mais recente e de configuração diferenciada (escala de diferencial semântico por adjetivos) é a Family Satisfaction by Adjectives Scale (FSAS) (Barraca et al., 2000), que contém 27 itens avaliando o grau de satisfação com a família a partir de uma ponderação de emoções positivas e negativas emitidas pelas pessoas quando estão em contato com sua família. Sua estrutura é configurada em escala de diferencial semântico onde são alocados adjetivos opostos e, as pessoas respondem ao seguinte estímulo "Quando estou com minha família, sinto-me...”. Uma das limitações dessa escala é que seus achados advêm de pesquisas realizadas apenas com universitários, além de medir exclusivamente o componente afetivo da satisfação familiar (Zabriskie, \& Ward, 2013).

A Family Satisfaction Scale (Carver, \& Jones, 1992) é composta por 20 itens e avalia o construto como um julgamento subjetivo, analisa especificamente a satisfação com a família de origem, o que é apontado como uma de suas limitações (Zabriskie, \& Ward, 2013), ou seja, cada membro vai ter como referência a sua família de origem (pais, mães, por exemplo) e não a atual (esposo(a), filhos).

Partindo de uma perspectiva global da avaliação do construto em questão, foram elaborados alguns instrumentos os quais permitiram que os indivíduos refletissem sobre diferentes áreas de sua vida familiar, seguindo o seu próprio arcabouço de valores e critérios. Entendese que, dessa forma, foi possível obter uma medida mais clara da satisfação familiar, ao invés de utilizar uma lista de domínios já pré-definida (Ward et al., 2009).

Seguindo essa perspectiva, Zabriskie e McCormick (2003) propuseram a Satisfaction With Family Life Scale (SWFL) que avalia o grau de satisfação que o sujeito julga ter em sua vida familiar, baseando-se nos seus próprios critérios. Por esse motivo, é considerada uma proposta de avaliação global, ou seja, cognitiva (julgamento avaliativo) e subjetiva (os próprios critérios da pessoa). Além disso, baseia-se na Satisfaction With Life Scale (SWLS) (Diener et al., 1985), medida amplamente utilizada e aceita na literatura em âmbito nacional e internacional, a exemplo de países, como França (Blais et al., 1989), China (Bai et al., 2011) e Noruega (Clench-Aas et al., 2011). A SWLS tem demonstrado boas qualidades psicométricas de validade $\mathrm{e}$ fidedignidade, com índices de confiabilidade superiores a 0,70 e adequação na replicação de sua estrutura unifatorial (Bedin, \& Sarriera, 2014; Gana et al., 2016; Pavot, $\&$ Diener, 2008).

A escala SWFL é composta de cinco itens, os quais são respondidos em uma escala tipo Likert de sete pontos, que variam de 1 (Discordo totalmente) a 7 (Concordo totalmente), a exemplo do item 5: "Se eu pudesse viver minha vida de novo, não mudaria quase nada que eu vivi com minha família”. Observa-se que o instrumento tem apresentado bons índices de validade e fidedignidade em amostras de outros países. Na pesquisa de Bernal e Arocena (2014), por exemplo, realizada no México, com uma amostra de 1.395 pessoas com idades entre 11 e 19 anos, os autores encontraram um alfa de Cronbach de 0,86 e uma estrutura unifatorial.

Acrescenta-se ainda que no estudo de Costa e Neto (2019), também foi identificada uma estrutura unifatorial da medida, com índice de precisão e ajuste satisfatórios (alfa de Cronbach $=0,92$, GFI $=0,97, \mathrm{CFI}=0,99$, SRMR $=0,02$, RMSEA $=0,06)$. Neste último, foi atestada a validade convergente e discriminante da escala SWFL, relacionando-a, respectivamente, a uma medida que mensura o mesmo construto e, a uma escala de solidão. Os resultados indicaram uma correlação positiva e significativa com a primeira $(r=0,64)$ e negativa com a segunda $(r=-0,26)$.

Pode-se destacar como vantagens do uso desse instrumento, os seguintes aspectos: (a) a avaliação da satisfação familiar num enfoque global, cognitivo e subjetivo, aspecto não identificado nos demais instrumentos mencionados; (b) apresentar bons índices de validade e precisão e, (c) ser de fácil aplicação, tendo em vista que é um instrumento curto, com apenas cinco itens, e parcimonioso.

Assim, como a satisfação com a vida, acredita-se que a satisfação familiar deve partir da avaliação do indivíduo acerca das diversas áreas que ele tem por base como importantes para sua vida familiar, para isso, utilizam-se de critérios subjetivos, fazendo um julgamento avaliativo da sua vida familiar.

O presente estudo torna-se relevante em função da necessidade de se ter, no contexto brasileiro, um instrumento válido e preciso para avaliação da satisfação familiar, pois, munido desses recursos, poder-se-á avaliar a relação e/ou as contribuições dela para a saúde mental, condutas de risco, engajamento escolar, comportamentos de ajuda, gestão de conflitos interpessoais, dentre outros comportamentos (Silva et al., 2017; Woyciekoski et al., 2014). Ademais, espera-se contribuir 
com a expansão da produção científica nessa temática que é pouco explorada no país.

Dito isso, o presente estudo tem por objetivo adaptar a Satisfaction With Family Life (SWFL) para o português-brasileiro, verificando evidências de validade fatorial e consistência interna, além da adequação de seu modelo unifatorial. Para alcançar os objetivos, a pesquisa foi dividida em dois estudos empíricos, os quais serão apresentados a seguir:

\section{Estudo 1. Adaptação e Evidências Preliminares de Validade e Precisão da SWFL}

Esse estudo teve como objetivo adaptar a SWFL para o português-brasileiro, reunindo evidências de validade e precisão da medida, além de verificar as características de dificuldade e discriminação de seus itens. Trata-se de uma análise preliminar dessa medida, a fim de verificar sua estrutura fatorial na representação do construto Satisfação familiar.

\section{Método}

\section{Participantes}

Contou-se com 216 pessoas da população geral da Região Nordeste do Brasil, selecionadas por meio de uma amostragem não probabilística, por conveniência, os quais concordaram em participar voluntariamente do estudo. Utilizou-se como critério de inclusão ser maior de 18 anos e ter escolaridade igual ou superior ao ensino fundamental incompleto. A idade dos participantes variou entre 18-92 anos $(M=38,43 ; D P=15,90)$, sendo a maioria do sexo feminino $(71,6 \%)$, casados $(49,1 \%)$, de religião protestante $(67,3 \%)$ e com ensino superior completo $(26,9 \%)$.

\section{Instrumentos}

Os seguintes instrumentos foram aplicados aos participantes:

Satisfaction With Family Life Scale (SWFLS) (Zabriskie, \& McCormick, 2003). É composta de cinco itens, adaptados da Satisfaction with Life Scale (SWLS) de Diener et al. (1985). Avalia o grau de satisfação que o indivíduo julga ter na vida familiar a partir de seus próprios critérios. Os itens (a exemplo do item 1: "Na maioria dos aspectos, minha vida familiar é próxima do meu ideal; item 3: Estou satisfeito(a) com minha vida familiar)" são respondidos numa escala tipo Likert de 7 pontos que vai de 1 (Discordo totalmente) a 7 (Concordo totalmente). O instrumento é unifatorial e reportou uma confiabilidade de 0,93 (teste-reteste: $r=0,89$ ).

Questionário sociodemográfico: constituído de questões direcionadas à caracterização da amostra, tais como: idade, sexo, escolaridade, estado civil e religião.

\section{Procedimentos}

A priori foi realizada a tradução da língua original (inglês) para o português brasileiro, mediante tradutores bilíngues independentes, a fim de sanar quaisquer vieses de ordem linguística, psicológica, cultural ou de compreensão. De posse das duas traduções, foram feitas comparações entre ambas, a fim de identificar discrepâncias, para se chegar a uma única versão. Esta foi avaliada pelos autores em termos de equivalência semântica de idioma e de conceito entre ela e o instrumento original.

Vencida essa etapa, a última versão do instrumento passou pela avaliação de experts em avaliação psicológica, os quais verificaram questões como instruções do instrumento e adequação das expressões utilizadas nos itens para diferentes regiões e público-alvo. Em seguida, a versão resultante desse processo foi apresentada a um estrato da população, que contou com 10 pessoas, a fim de avaliar a compreensão dos itens, das instruções e da escala de respostas. Foi solicitado que respondessem ao instrumento e que, ao final, indicassem modificações, caso não estivesse claro. Não sendo apontada nenhuma sugestão pelos respondentes, procedeu-se um estudo piloto com indivíduos da população geral. Estes não relataram quaisquer problemas com os itens, instruções ou escala de respostas. Assim, chegou-se à versão final utilizada na presente pesquisa. Todos os passos acima descritos são etapas propostas por Borsa et al. (2012) e Pasquali (2010) para adaptação de instrumentos psicológicos entre culturas. Após esse cuidadoso procedimento, verificou-se que a versão em português refletia a versão original.

O instrumento foi aplicado aos participantes de forma presencial (lápis e papel) e online. A literatura aponta evidências de que os dados provenientes de ambas as estratégias de coleta de dados são equivalentes (Brock et al., 2012). No momento da aplicação do instrumento, eram apresentados os objetivos da pesquisa e o caráter voluntário da participação, que levou em média $10 \mathrm{mi}-$ nutos. Atentou-se para todos os requisitos éticos exigidos às pesquisas com seres humanos, garantido o anonimato e o caráter voluntário da participação, conforme o disposto nas Resoluções 466/2012 e 510/2016 do Conselho Nacional de Saúde, tendo sido aprovado pelo Comitê de Ética da Universidade Federal da Paraíba através da Plataforma Brasil (CAAE: 79837417.3.0000.5188). A participação foi confirmada mediante assinatura do Termo de Consentimento Livre e Esclarecido pelo sujeito.

\section{Análises de Dados}

Foi utilizado o SPSS (versão 21) para calcular estatísticas descritivas (média, desvio padrão) e estimativas de frequências a fim de caracterizar a amostra. Para verificar a validade da SWFLS, procedeu-se a avaliação da sua estrutura fatorial. Para isso investigou-se a dimensionalidade a partir de uma análise fatorial exploratória. Como a escala não apresenta uma distribuição normal e tem uma configuração tipo Likert com categorias ordenadas, utilizou-se o software Factor 9.2 (Lorenzo-Seva, \& Ferrando, 2013), método Hull Comparative Fit Index para retenção 
fatorial (CFI; Lorenzo-Seva et al., 2011) e Weighted Least Squares Mean-and Variance-adjusted (WLSMV) como método de extração de fatores, com correlações policóricas. Conforme afirma Lorenzo-Seva et al. (2011), o método Hull é um dos mais indicados na estimação da dimensionalidade de um conjunto de itens.

Outra evidência da validade da medida adaptada é a precisão, cuja verificação, neste estudo, foi realizada por meio dos índices alfa de Cronbach e ômega de McDonald (Urbina, 2007). A dificuldade e discriminação dos itens também foi analisada a partir dos parâmetros a e b (thresholds).

\section{Resultados}

A fim de realizar a análise fatorial exploratória, foram obtidos os seguintes índices para verificar a adequação da matriz de correlação: Kaiser-Meyer-Olkin $(\mathrm{KMO})=0,88$ e teste de esfericidade de Bartlett $=749.4$ (10); $p<0,001$, evidenciando que a matriz de correlações se mostrou adequada. Verificando-se a estrutura fatorial da medida pelo método Hull Comparative Fit Index (CFI; Lorenzo-Seva et al., 2011) emergiu uma solução unifatorial, fornecendo um índice de ajuste Global Fit Index $(\mathrm{GFI})=0,99$, que explica $75 \%$ da variância total dos itens. A seguir (Tabela 1) são apresentadas as cargas fatoriais e a comunalidade dos itens, além dos índices de consistência interna da escala.

Em suma, os achados confirmam a estrutura unifatorial, fornecendo resultados satisfatórios que atestam a validade de construto do SWFLS. Todas as cargas fatoriais apresentaram valores superiores a 0,30 no fator, atendendo ao critério de saturação mínima sugerido na literatura (Pasquali, 2010). Os cinco itens descritos na Tabela 1, adaptados ao português-brasileiro, compõem a estrutura final do instrumento, permanecendo a mesma da medida original. A maior saturação foi encontrada no item $1=0,89$ ("Na maioria dos aspectos, minha vida familiar é próxima do meu ideal”), e, a menor, no item $5=$ 0,74 ("Se eu pudesse viver minha vida de novo, não mudaria quase nada que vivi com minha família"). Ademais, os índices refletidos nos valores do $\Omega$ de McDonald $(0,90)$ e $\alpha$ de Cronbach $(0,89)$ sugerem que o instrumento apresenta consistência interna satisfatória, o que permite dizer, portanto, que é unifatorial e denomina-se de Satisfação Familiar.

Tabela 1

Estrutura Fatorial da Satisfaction With Family Life Scale (SWFL)

\begin{tabular}{|c|c|c|c|c|}
\hline Itens & Fator & $h^{2}$ & a & $b$ \\
\hline 1. Na maioria dos aspectos, minha vida familiar é próxima do meu ideal. & $0,89^{*}$ & 0,79 & 1,90 & $-1,13$ \\
\hline 2. As condições da minha vida familiar são excelentes. & $0,83^{*}$ & 0,69 & 1,41 & $-1,21$ \\
\hline 3. Estou satisfeito(a) com minha vida familiar. & $0,85^{*}$ & 0,72 & 1,64 & $-1,39$ \\
\hline 4. Eu obtive sucesso em grande parte das coisas importantes que eu queria na vida familiar. & $0,85^{*}$ & 0,71 & 1,64 & $-1,15$ \\
\hline 5. Se eu pudesse viver minha vida de novo, não mudaria quase nada que vivi com minha família. & $0,74^{*}$ & 0,55 & 1,07 & $-1,13$ \\
\hline Quantidade de itens & 5 & & & \\
\hline Variância explicada (\%) & $75 \%$ & & & \\
\hline Valor próprio & 3,44 & & & \\
\hline$\Omega$ de McDonald & 0,90 & & & \\
\hline$\alpha$ de Cronbach & 0,89 & & & \\
\hline
\end{tabular}

Nota. ${ }^{~}$ carga fatorial considerada satisfatória, isto é, $>|0,30| . h^{2}=$ comunalidade; $a$ - parâmetro de discriminação do item; $b$ - parâmetro de dificuldade do item

Os parâmetros de dificuldade (b) e discriminação (a) dos itens foram avaliados obtendo-se os seguintes valores, apresentados na Tabela 1. Pode-se observar, a partir dos resultados, que todos os itens apresentam valores altos no parâmetro a, acima de 1,70, indicando que estes têm a capacidade de discriminar pessoas em dois grupos: os que possuem níveis de satisfação familiar (habilidade) abaixo do valor do parâmetro b e os que possuem habilidades acima do parâmetro b.

Já no que se refere à dificuldade dos itens, os índices variaram entre -1,39 (item 3) e -1,13 (itens 1 e 5). O item 3 "Estou satisfeito(a) com minha vida familiar " foi o mais fácil de ser respondido, enquanto os itens 1 e 5 "Na maioria dos aspectos, minha vida familiar é próxima do meu ideal" e "Se eu pudesse viver minha vida de novo, não mudaria quase nada que vivi com minha família”, respectivamente, foram o que ofereceram maior dificuldade de resolução.

\section{Estudo 2. Evidências Adicionais de Estrutura Interna da SWFLS}

Neste estudo, foram realizadas análises de natureza confirmatória, a fim de adicionar evidências aos achados do estudo anterior. Esse recurso fornece conclusões mais substanciais à validade fatorial da medida, permitindo verificar o quanto ela se ajusta aos dados empíricos. 


\section{Método}

\section{Participantes}

Contou-se com 214 indivíduos da população geral, domiciliados na Região Nordeste do Brasil, com idades variando entre 18 e 77 anos $(M=29,8 ; D P=11,65)$, sendo a maioria do sexo feminino $(69,6 \%)$ com ensino superior incompleto $(40,7 \%)$, solteira $(64 \%)$ e de religião protestante $(45,3 \%)$.

\section{Instrumentos}

Os participantes responderam a Satisfaction with Family Life Scale (SWFLS) (Zabriskie, \& McCormick, 2003), adaptada e validada no Estudo 1, bem como o questionário sociodemográfico contendo as mesmas perguntas já referidas.

\section{Procedimentos}

A aplicação dos instrumentos ocorreu de forma semelhante ao Estudo 1, sendo respeitadas todas as instruções elencadas nas Resoluções 466/12 e 510/2016 do Conselho Nacional de Saúde.

\section{Análises de Dados}

Utilizou-se o software R, por meio do pacote Lavaan (Rossel, 2012), para execução da análise fatorial confirmatória (AFC) com o uso do método de estimação Weighted Least Squares Mean-and Variance-adjusted (WLSMV). Para verificar se o modelo era adequado, recorreu-se aos indicadores: Comparative Fit Index (CFI), índice que faz uma comparação entre a matriz de covariância predita pelo modelo e a matriz observada. Espera-se valores iguais ou superiores a 0,90 para atestar um modelo ajustado; Tucker-Lewis Index (TLI), nesse índice os valores podem variar entre 0 e 1 , são considerados adequados quando superiores a 0,90; e Root-Mean-Square Error of Approximation (RMSEA) com intervalo de confiança de 90\% (IC90\%), está relacionado ao ajuste do modelo, assim valores altos evidenciam um modelo não ajustado. Sugere-se, portanto, valores até 0,10 (Byrne, 2010; Tabachnick, \& Fidell, 2013).

\section{Resultados}

Considerando os resultados do instrumento original e da validação realizada no Estudo 1, buscou-se testar o modelo unifatorial da SWFLS (versão adaptada e validada no Estudo 01) com seus cinco itens.

Realizando uma análise fatorial confirmatória, o modelo forneceu um índice GFI de 0,99, TLI de 0,99, RMSEA $=0,00$ (IC $90 \% 0,01-0,07)$. As cargas fatoriais dos itens $(\lambda)$ variaram entre 0,49 (Item 5 . Se eu pudesse viver minha vida de novo, não mudaria quase nada que vivi com minha família) e 0,71 (Item 3. Estou satisfeito(a) com minha vida familiar). Como todos os pesos dos Lambdas $(\lambda)$ foram positivos e diferentes de zero $(z>1,96, p<0,001)$, pode-se afirmar que o fator prediz as respostas aos itens de modo satisfatório. Esses achados corroboram a estrutura original, ou seja, há evidências de que a SWFLS é adequadamente representada por seus cinco itens e as informações estatísticas atestam sua unidimensionalidade. Os indicadores de consistência interna apresentaram-se adequados: $\alpha$ de Cronbach $=0,89$ e $\Omega$ de McDonald=0,90.

Figura 1

Análise fatorial confirmatória da SWFLS

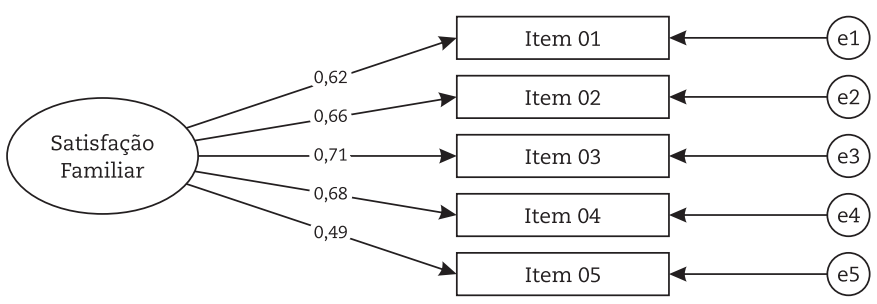

\section{Discussão Geral}

Acredita-se que os objetivos dos dois estudos foram alcançados, a saber: reunir evidências psicométricas da SWFLS, verificando sua validade fatorial, consistência interna e propriedades de dificuldade e discriminação dos itens; além de analisar o ajustamento do modelo fatorial hipotetizado (unifatorial). A seguir serão discutidos os principais achados da pesquisa.

O processo de validação da SWFLS para o português-brasileiro ocorreu mediante realização de uma análise fatorial exploratória pelo método Hull. Um único fator emergiu da solução e corresponde a dimensão Satisfação familiar. Esperava-se que os itens se agrupassem num único fator, conforme resultados da medida original e estrutura de outros instrumentos que medem o mesmo construto (Barraca et al., 2000; Carver, \& Jones, 1992; Zabriskie, \& Ward, 2013), o que ocorreu de modo total. $\mathrm{O}$ fator extraído da análise foi considerado válido por apresentar itens com boas cargas fatoriais, aceitáveis 
para estudos exploratórios (Hair et al., 2009). O mesmo resultado foi encontrado no estudo de Bernal e Arocena (2014), cuja estrutura também resultou em unifatorial, com confiabilidade ( $\alpha$ de Cronbach) igual a 0,86, explicando $55,80 \%$ da variância. Ademais, os índices de dificuldade e discriminação dos itens foram considerados satisfatórios, reunindo evidências da qualidade da medida.

No Estudo 2, o modelo unifatorial foi corroborado, o qual se mostrou ajustado, a partir dos parâmetros utilizados na análise confirmatória (GFI, TLI, RMSEA). Esse tipo de análise confere nova clareza da validade da estrutura interna da medida, pois ajusta o modelo aos dados empíricos. No estudo de construção e validação inicial da SWFLS, não foram realizadas análises confirmatórias. Em contrapartida Bernal e Arocena (2014) efetuaram essa análise e seus achados indicaram bons ajustes do modelo unifatorial $(\mathrm{GFI}=0,993$, RMSEA $=0,047, \mathrm{CFI}=0,994$; cargas fatoriais entre 0,59 e 0,85$)$.

Diante desses resultados, pode-se afirmar que o instrumento tem qualidades psicométricas satisfatórias para avaliação da satisfação com a vida familiar no âmbito da pesquisa. Conforme corroborado em estudos anteriores, fica manifesto que a SWFLS possui evidências para sustentar sua estrutura unidimensional.

Não obstante, como limitação do estudo, pode-se indicar a regionalização da amostra, pois origina-se exclusivamente do Nordeste do país. Ademais, foi utilizada amostragem não probabilística (por conveniência) na coleta de dados, o que pode interferir na generalização dos resultados, todavia, tal fato não foi intencionado pelos autores. A desejabilidade social também é um aspecto a ser considerado como limitador, uma vez que a medida avaliada é de autorrelato. Esse tipo de instrumento pode levar o participante a respondê-lo de forma socialmente favorável e não com o que representa sua opinião ou comportamento de fato (Tracey, 2016). Ainda quanto as limitações do estudo, pontua-se que não foi realizado qualquer controle sobre dados sociodemográficos, sendo a maioria da amostra composta por mulheres e com nível educacional superior (completo e incompleto), dado que pode ser mais bem calibrado em pesquisas futuras.

Embora os estudos na área sejam recentes, a satisfação familiar figura como um objeto de estudo essencial a ser considerado em diversas pesquisas, principalmente aquelas que lidam diretamente com as contribuições da família nas atitudes e comportamentos de seus descendentes (Scorsolini-Comin et al., 2015; Scorsolini-Comin et al., 2016). Frente a isso, é de suma importância que investigações como esta levem pesquisadores a refletirem sobre o tema, passando a considerar os aspectos positivos das pessoas em suas investigações (Hutz et al., 2014).

Em conclusão, reafirma-se a importância de investigações com medidas direcionadas à satisfação familiar, frente a carência dessas ferramentas, bem como da realização de estudos na área no contexto brasileiro. Assim, recomenda-se o desenvolvimento de novas pesquisas, utilizando-se diferentes análises, como validade convergente e discriminante, em amostras das demais regiões do país a fim de somar aos achados de validade da SWFLS.

\section{Agradecimentos}

O presente artigo contou com apoio da CAPES que concedeu bolsas de doutorado à primeira autora. Aproveitamos para demonstrar nossa gratidão à essa instituição.

\section{Financiamento}

Todas as fontes de financiamento para elaboração e produção do estudo (coleta, análise e interpretação dos dados, bem como, escrita dos resultados no presente no manuscrito) foram fornecidas pelo projeto de pesquisa "Código de Financiamento 001 - CAPES.

\section{Contribuições dos autores}

Declaramos que todos os autores participaram da elaboração do manuscrito. Especificamente, o(s) autor(es) Luize Anny Guimarães Amorim participou(ram) da redação inicial do estudo - conceitualização, investigação, visualização, o(s) autor(es) Luize Anny Guimarães Amorim participou(ram) da análise dos dados, e o(s) autor(es) Patrícia Nunes da Fonseca participou(ram) da redação final do trabalho - revisão e edição.

\section{Disponibilidade dos dados e materiais}

Todos os dados e sintaxes gerados e analisados durante esta pesquisa serão tratados com total sigilo devido às exigências do Comitê de Ética em Pesquisa em Seres Humanos. Porém, o conjunto de dados e sintaxes que apoiam as conclusões deste artigo estão disponíveis mediante razoável solicitação ao autor principal do estudo.

\section{Conflito de interesses}

Os autores declaram que não há conflitos de interesses.

\section{Referências}

Andrews, I. M., \& Withey, S. B. (1976). Social Indicators of Wellbeing. American Perceptions of Life Quality. Plenum.

Bai, X., Wu, C., Zheng, R., \& Ren, X. (2011). The Psychometric Evaluation of the Satisfaction With Life Scale Using a Nationally Representative Sample of China. Journal of Happiness Studies, 12(2), 183-197. http://dx.doi.org/10.1007/s10902-010-9186-x 
Barraca, J., Yarto, L. L., \& Olea, J. (2000). Psychometric Properties of a New Family Life Satisfaction Scale. European Journal of Psychological Assessment, 16(2), 98-106. http://dx.doi.org/10.1027//1015-5759.16.2.98

Bedin, L. M., \& Sarriera, J. C. (2014). Propriedades Psicométricas das Escalas de Bem-estar: PWI, SWLS, BMSLSS e CAS. Avaliação Psicológica, 13(2), 213-225. http://pepsic.bvsalud.org/scielo.php?script=sci arttext\&pid=S1677-04712014000200009

Bernal, A. C. A. L., \& Arocena, F. A. L. (2014). Established Satisfaction With Family Life Scale Factor in Middle School and High School Adolescents. Psicogente, 17(31), 226-240. http://dx.doi.org/10.17081/psico.17.31.433

Blais, M. R., Vallerand, R. J., Pelletier, L. G., \& Brière, N. M. (1989). Léchelle De Satisfaction De Vie: Validation Canadienne-Française Du "Satisfaction With Life Scale". Canadian Journal of Behavioural Science, 21(2), 210-223. http://dx.doi.org/10.1037/h0079854

Borsa, J. C., Damásio, B. F., \& Bandeira, D. R. (2012). Adaptação e Validação de Instrumentos Psicológicos entre Culturas: Algumas Considerações. Paidéia (Ribeirão Preto), 22(53), 423-432. http://dx.doi.org/10.1590/S0103-863X2012000300014.

Brasil (1988). Constituição da República Federativa do Brasil de 1988. Presidência da República, Brasília. http://www.planalto.gov.br/ccivil_03/ Constituicao/Constituicao.htm

Brock, R. L., Barry, R. A., Lawrence, E., Dey, J., \& Rolffs, J. (2012). Internet Administration of Paper-and-pencil Questionnaires Used in Couple Research: Assessing Psychometric Equivalence. Assessment, 19(2), 226-242. http://dx.doi.org/10.1177/1073191110382850

Byrne, B. M. (2010). Structural Equation Modeling with Amos: Basic Concepts, Applications, and Programmimg (2a ed.). Routledge.

Campbell, A., Converse, P. E., \& Rodgers, W. L. (1976). The Quality of American Life: Perceptions, Assessments and Satisfactions. Russell Sage Foundation.

Carver, M. D., \& Jones, W. H. (1992). The Family Satisfaction Scale. Social Behavior \& Personality: An International Journal, 20(2), 71-82. http:// dx.doi.org/10.2224/sbp.1992.20.2.71.

Clench-Aas, J., Nes, R. B., Dalgard, O. S., \& Aarø, L. E. (2011). Dimensionality and measurement invariance in the Satisfaction With Life Scale in Norway. Quality of Life Research, 20(8), 1307-1317. http://dx.doi.org/10.1007/s11136-011-9859-x

Costa, M. P., \& Neto, F. (2019). Psychometric Evaluation of the Portuguese Satisfaction With Family Life Scale. Measurement Instruments Social Sciences, 1, 1-8. http://dx.doi.org/10.1186/s42409-019-0009-5

Diener, E., Emmons, R. A., Larsen, R. J., \& Griffin, S. (1985). The Satisfaction With Life Scale. Journal of Personality Assessment, $49(1), 71-75$. http://dx.doi.org/10.1207/s15327752jpa4901_13

Gana, K., Broc, G., Saada, Y., Amieva, H., \& Quintard, B. (2016) Subjective Wellbeing and Longevity: Findings from a 22-Year Cohort Study. Journal of Psychosomatic Research, 85, 28-34. http://dx.doi.org/10.1016/j.jpsychores.2016.04.004

Grilo, I., \& Major, S. (2015). Qualidade de Vida Familiar, Satisfação com a Vida e Apoio Social Percebido na Deficiência Visual. Temas em Psicologia, 23(2), 327-339. http://dx.doi.org/10.9788/TP2015.2-07

Guerney, B. G., Jr. (1977). Relationship Enhancement: Skill Training Programs for Therapy, Problem Prevention, and Enrichment. Jossey-Bass.

Hair, J. F., Black, W. C., Babin, B. J., Anderson, R. E., \& Tatham, R. L. (2009). Análise Multivariada dos Dados (6 $6^{\mathrm{a}}$ ed.). Bookman.

Hutz, C., Midgett, A., Pacico, J., Bastianello, M., \& Zanon, C. (2014). The Relationship of Hope, Optimism, Self-esteem, Subjective Wellbeing, and Personality in Brazilians and Americans. Psychology, 5, 514-522. http://dx.doi.org/10.4236/psych.2014.56061

Lisboa, C., Wendt, G. W., Neufeld, C. B., \& Matos, M. G. (2014). Satisfação com a Vida e com a Família e Violência Interpessoal na Adolescência. Revista Brasileira de Terapias Cognitivas, 10(1), 19-28. http://dx.doi.org/10.5935/1808-5687.20140004

Lorenzo-Seva, U., \& Ferrando, P. J. (2013). FACTOR 9.2: A Comprehensive Program for Fitting Exploratory and Semiconfirmatory Factor Analysis and IRT Models. Applied Psychological Measurement, 37(6), 497-498. http://dx.doi.org/10.1177/0146621613487794

Lorenzo-Seva, U., Timmerman, M. E., \& Kiers, H. A. L. (2011). The Hull Method for Selecting the Number of Common Factors. Multivariate Behavioral Research, 46(2), 340-364. http://dx.doi.org/10.1080/00273171.2011.564527

McCollum, E. E., Schumm, W. R., \& Russell, C. S. (1988). Reliability and Validity of the Kansas Family Life Satisfaction Scale in a Predominantly Middle-Aged Sample. Psychological Reports, 62(1), 95-98. http://dx.doi.org/10.2466/pr0.1988.62.1.95

Olson, D. H. (1979). Circumplex Model of Marital and Family Systems. I. Cohesion and Adaptability Dimensions, Family Types, and Clinical Applications. Family Process, 18(1), 3-28. http://dx.doi.org/10.1111/j.1545-5300.1979.00003.x

Olson, D. H. (2004). Circumplex Model VII: Validation Studies and FACES III. Family Process, 25, 337-351. http://dx.doi.org/10.1111/ j.1545-5300.1986.00337.x

Olson, D. H. (2011). FACES IV and the Circumplex Model: Validation Study.Journal Marital Family, 37(1), 64-80. http://dx.doi.org/10.1111/ j.1752-0606.2009.00175.x

Olson, D. H., \& Wilson, M. (1982). Family Inventories: Inventories Used in a National Survey of Families Across the Family Life Cycle. In D. H. Olson, H. I. McCubbin, H. Barnes, A. Larsen, M. Muxen, \& M. Wilson (Eds.), Family Social Science (pp. 25-31). University of Minnesota.

Olson, D. H., Portner, J., \& Lavee, Y. (1985). FACES III. Family Social Science. University of Minnesota.

Pasquali, L. (2010). Instrumentação Psicológica: Fundamentos e Práticas. Artmed.

Pavot, W., \& Diener, E. (2008) The Satisfaction With Life Scale and The Emerging Construct of Life Satisfaction. The Journal of Positive Psychology, 3(2), 137-152. http://dx.doi.org/10.1080/17439760701756946

Rossel, Y. (2012). lavaan: An R Package for Structural Equation Modeling. Journal of Statistical Software, 48(2), 1-36. http://dx.doi.org/10.18637/ jss.v048.i02

Santos, P. L., Bazon, M. R., \& Carvalho, A. M. P. (2017). Family Adaptability and Cohesion Evaluation Scale IV (FACES IV): Adaptação Brasileira. Avaliação Psicológica, 16(2), 120-127. http://dx.doi.org/10.15689/AP.2017.1602.01

Schumm, W. R., McCollum, E. E., Bugaighis, M. A., Jurich, A. P., \& Bollman, S. R. (1986). Characteristics of the Kansas Family Life Satisfaction Scale in a Regional Sample. Psychological Reports, 58(3), 975-980. http://dx.doi.org/10.2466/pr0.1986.58.3.975

Scorsolini-Comin, F., Fontaine, A. M. G. V., Barroso, S. M., \& Santos, M. A. (2016). Fatores Associados ao Bem-estar Subjetivo em Pessoas Casadas e Solteiras. Estudos de Psicologia (Campinas), 33(2), 313-324. http://dx.doi.org/10.1590/1982-02752016000200013

Scorsolini-Comin, F., Fontaine, A. M. G. V., Barroso, S. M., \& Santos, M. A. (2015). Relações entre Conjugalidade dos Pais, Conjugalidade dos Filhos e Bem-estar Subjetivo. Psico-USF, 20(3), 481- 92. http://dx.doi.org/10.1590/1413-82712015200310

Seligman, \& Csikszentmihalyi (2000). Positive Psychology: An introduction. American Psychologist, 55(1), 5-14. http://dx.doi.org/10.1037/0003066X.55.1.5

Shin, D. C., \& Johnson, D. M. (1978). Avowed Happiness as an Overall Assessment of the Quality of Life. Social Indicators Research, 5(1-4), 475-492. http://dx.doi.org/10.1007/BF00352944 
Silva, D. G., Giordani, J. P., \& Dell’ Aglio, D. D. (2017). Relações entre Satisfação com a Vida, com a Família e com as Amizades e Religiosidade na Adolescência. Estudos Interdisciplinares em Psicologia, 8(1), 38-54. http://dx.doi.org/10.5433/2236-6407.2017v8n1p38

Tabachnick, B. G., \& Fidell, L. S. (2013). Using Multivariate Statistics (6th ed.). Allyn and Bacon.

Tracey, T. J. G. (2016). A Note on Socially Desirable Responding. Journal of Counseling Psychology, 63(2), 224-32. http://dx.doi.org/10.1037/ cou0000135

Urbina, S. (2007). Fundamentos da Testagem Psicológica. Artmed.

Walsh, F. (2016). Diversidade e Complexidade nas Famílias do Século XXI. Em F. Walsh (Ed.), Processos Normativos da Família: Diversidade e Complexidade (pp. 3-27). Artmed.

Ward, P., Lundberg, N., Zabriskie, R., \& Barrett, K. (2009). Measuring marital satisfaction: a comparison of the revised dyadic adjustment scale and the satisfaction with married life scale. Marriage and Family Review, 45(4), 412- 429. http://dx.doi.org/10.1080/01494920902828219

Weiting, N. (2017). Extending tradicional psychological disciplines to positive psychological: a view from subjective well-being. Journal Happiness Studies, 18(5), 1553-1571. http://dx.doi.org/10.1007/s10902-016-9782-5

Woyciekoski, C., Natividade, J. C., \& Hutz, C. S. (2014). As contribuições da personalidade e dos eventos de vida para o bem-estar subjetivo. Psicologia: Teoria e Pesquisa, 30(4), 401-409. https://www.scielo.br/j/ptp/a/HqWb5vDtJ6sCJnTy46XMqNn/?lang=pt\&format=pdf

Zabriskie, R. B., \& McCormick, B. P. (2003). Parent and child perspectives of family leisure involvement and satisfaction with family life. Journal of Leisure Research, 35(2), 163-189. http://dx.doi.org/10.1080/00222216.2003.11949989

Zabriskie, R. B., \& Ward, P. J. (2013). Satisfaction with family life scale. Marriage E Family Review, 49(5), 446-463. http://dx.doi.org/10.1080 /01494929.2013.768321

\section{Sobre as autoras}

Luize Anny Guimarães Amorim é psicóloga (UFPB), doutora em Psicologia Social pela Universidade Federal da Paraíba. Atualmente é Professora no curso de Direito da Faculdade Três Marias e EESAP, localizadas na cidade de João Pessoa-PB e Guarabira-PB, respectivamente.

Patrícia Nunes da Fonseca é psicóloga (UFPB) e bacharel em Direito (Centro Universitário de João Pessoa), doutora em Psicologia Social pela Universidade Federal da Paraíba. Atualmente é Professora do Departamento de Psicologia (UFPB) e do Programa de Pósgraduação em Psicologia Social (UFPB).

\section{Como citar este artigo}

Amorim, L. A. G., \& Fonsêca, P. N. (2021). Satisfaction With Family Life Scale: adaptação e validação. Avaliação Psicológica, 20(3), 299-307. http://dx.doi.org/10.15689/ap.2021.2003.18386.04 